\section{Ein Jahr HeilberufeSCIENCE}

HeilberufeSCIENCE feiert mit der

Ausgabe 4/2009 den ersten Geburtstag. Jetzt existiert ein erster vollständiger Jahrgang im World Wide Web, der - nicht zu vergessen - auch noch um ein Sonderheft mit den Abstracts der Beiträge zum 7. Gesundheitspflegekongress vom 29.-31.10.2009 in Hamburg ergänzt wurde. Wenngleich in den vergangenen zwölf Monaten so manche redaktionellen Probleme im Hintergrund gelöst werden mussten, so erschienen doch alle Ausgaben termingerecht. Somit konnte HeilberufeSCIENCE Ihnen nicht nur Artikel aus den Gebieten Pflegemanagement, Pflegepädagogik, Pflegewissenschaften, Gerontologie, Altenpflege und Gesundheitswissenschaften/Public Health bieten, sondern auch verlässliche Veröffentlichungstermine für die Autorinnen und Autoren der Beiträge und natürlich für die Leserinnen und Leser.

Die nun "vorliegende“ Geburtstagsausgabiet "Patient" aus unterschiedlichen Blickrichtungen betrachten. Die Arbeit von Buttler zeigt, wie die Umsetzung des Expertenstandards ,,Sturzprophylaxe in der Pflege" in zwei stationären Pflegeeinrichtungen erfolgt und evaluiert werden konnten, so dass aus Bewohnern keine Sturzpatienten werden. Diese Ergebnisse bieten einen methodischen Ansatz für vergleichbare Einrichtungen. Einem anderen patientenorientierten Thema, aber gleichfalls methodisch ausgerichtet, widmet sich der Beitrag von Hoffmann et al. In dieser Arbeit zeigten die Autoren, wie wichtig es ist, be umfasst fünf Artikel, die das Themenge- bestimmte Qualitätsparameter und Qualitätszertifikate zur Grundlage für die Erstellung von Internetseiten für Patienten zu entwickeln, so dass sich Patienten bei der Auswahl geeigneter Informationen daran orientieren können. Die Untersuchung von Geisel-Marbeise und Stummer stellt ebenfalls den Patienten in den Mittelpunkt, indem sie das Phänomen der Adherence anhand von Apothekendaten bei Patienten mit Diabetes mellitus analysierten. Der Artikel von Enns et al. ist der zweite Teil eines Beitrags zur Alltagsbewältigung von älter werdenden Contergan-geschädigten Menschen (der erste erschien in HeilberufeSCIENCE 2/2009). Den Abschluss bildet die Arbeit von Flach und Laux zum Schmerzmanagement in der Pflege; sie befasst sich mit der Umsetzung des Expertenstandards in die elektronische Dokumentation pflegerischer Leistung, um eine optimale Versorgung von Schmerzpatienten zu erzielen.

Nun möchte ich Sie wieder einladen, diese vierte diesjährige Ausgabe von HeilberufeSCIENCE kritisch zu lesen und darüber die kühle Witterung zu vergessen. Außerdem wünsche ich Ihnen eine fröhliche Weihnachtszeit und alles Gute für das Jahr 2010 !

$\mathrm{Ihr}$

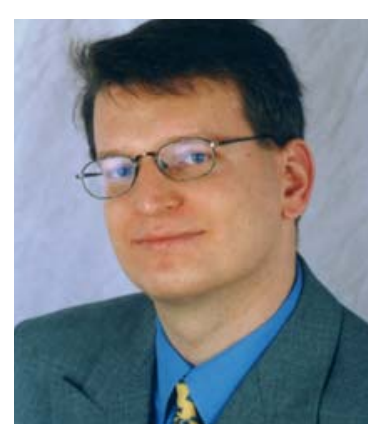

Prof. Dr. med. habil. Jörg Klewer, Zwickau

Schriftleitung

heilberufescience@springer.com

HeilberufeSCIENCE 2009; 2 (4): 93 DOI 10.1007/s00058-009-1619-8

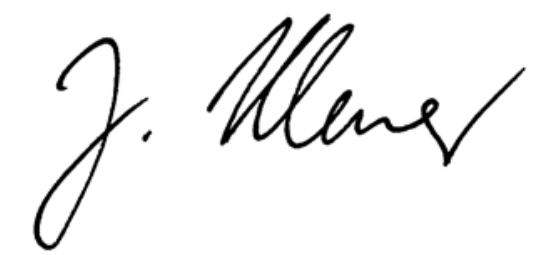

